\title{
Mutations in a member of the ADAMTS gene family cause thrombotic thrombocytopenic purpura
}

Gallia G. Levy ${ }^{\star}$, William C. Nichols $\dagger$, Eric C. Lian $\ddagger$, Tatiana Foroud $\S$, Jeanette N. McClintick $₫$, Beth M. McGee ${ }^{\star}$, Angela Y. Yang $^{\star}$, David R. Siemieniak ${ }^{\star}$, Kenneth R. Stark ${ }^{\star}$, Ralph Gruppo\|, Ravindra Sarodeg, Susan B. Shurin\#, Visalam Chandrasekaran $九$, Sally P. Stabler ${ }^{\star \star}$, Hernan Sabio $\dagger \dagger$, Eric E. Bouhassira $\neq \neq$, Jefferson D. Upshaw, Jr $\$ \$$, David Ginsburg \& Han-Mou Tsaill||

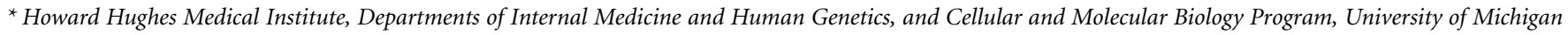
Medical Center, Ann Arbor, Michigan 48109, USA

$\dagger$ Division of Human Genetics, Children's Hospital Medical Center, Cincinnati, Ohio 45229, USA

$\$$ Hemophilia and Thrombosis Center and Sylvester Cancer Center, University of Miami and Veterans Affairs Medical Center, Miami, Florida 33136, USA

$\$$ Department of Medical and Molecular Genetics, Indiana University School of Medicine, Indianapolis, Indiana 46202, USA

II Division of Hematology/Oncology, Children's Hospital Medical Center, Cincinnati, Ohio 45229, USA

S Blood Bank, University of Texas Southwestern School of Medicine, Dallas, Texas 75390, USA

\# Department of Pediatrics, Rainbow Babies and Children's Hospital, and the Case Western Reserve University School of Medicine, Cleveland, Ohio 44106, USA

Blood Bank, Long Island Jewish Medical Center, and Albert Einstein College of Medicine, New Hyde Park, New York 11040, USA

** Department of Medicine, University of Colorado Health Sciences Center, Denver, Colorado 80262, USA

$\dagger \dagger$ Pediatric Hematology/Oncology, Medical College of Georgia, Augusta, Georgia 30912, USA

拉 Department of Medicine/Hematology and Department of Cell Biology, Albert Einstein College of Medicine, Bronx, New York 10461, USA

\$ The Memphis Cancer Center, Memphis, Tennessee 38119, USA

IIII Division of Hematology, Montefiore Medical Center, and Albert Einstein College of Medicine, Bronx, New York 10467, USA

Thrombotic thrombocytopenic purpura (TTP) is a life-threatening systemic illness of abrupt onset and unknown cause. Proteolysis of the blood-clotting protein von Willebrand factor (VWF) observed in normal plasma is decreased in TTP patients. However, the identity of the responsible protease and its role in the pathophysiology of TTP remain unknown. We performed genome-wide linkage analysis in four pedigrees of humans with congenital TTP and mapped the responsible genetic locus to chromosome 9q34. A predicted gene in the identifed interval corresponds to a segment of a much larger transcript, identifying a new member of the ADAMTS family of zinc metalloproteinase genes (ADAMTS13). Analysis of patients' genomic DNA identified 12 mutations in the ADAMTS13 gene, accounting for 14 of the 15 disease alleles studied. We show that deficiency of ADAMTS13 is the molecular mechanism responsible for TTP, and suggest that physiologic proteolysis of VWF and/or other ADAMTS13 substrates is required for normal vascular homeostasis.

TTP is characterized by intravascular destruction of erythrocytes and blood platelets resulting in anaemia and thrombocytopenia. Diffuse platelet-rich microthrombi are observed in small blood vessels of multiple organs, with the major complications including renal failure and neurologic dysfunction. In the related disorder, haemolytic uraemic syndrome (HUS), neurologic symptoms are less evident and renal failure is more prominent. HUS is most frequently observed in young children, usually following infection with a specific Shiga-toxin-producing strain of Escherichia coli. Although TTP-like disorders have also been associated with various medications, bone marrow transplantation, pregnancy, human immunodeficiency virus (HIV) infection, and autoimmune disease, most cases appear sporadically, without an obvious precipitating event. A subset of patients from these sporadic cases experience a chronic relapsing course. Rare familial cases of TTP have also been reported, generally associated with neonatal onset and frequent relapses (OMIM (http://www.ncbi.nlm.nih.gov/Omim) (accession numbers 274150 and 276850) ${ }^{1,2}$.

Before the development of modern treatment protocols consisting of fresh-frozen plasma infusion with or without plasma exchange, fatality during an acute episode of TTP was $>90 \%$ (refs 2 and 3). Although treatment outcome has improved significantly, the molecular pathogenesis of TTP is still unknown and the specific plasma factor(s) responsible for the acute onset of this disease, or recovery after treatment, remains to be identified. Unusually large multimeric forms of the platelet-adhesive blood-coagulation protein VWF have been observed in the plasma of TTP patients and are proposed to have a pathogenic role in the formation of the microvascular VWF- and platelet-rich thrombi characteristic of this disorder ${ }^{4}$. Consistent with this hypothesis, a proteolytic activity that degrades large VWF multimers to smaller sizes ${ }^{5,6}$ has been shown to be decreased in the plasma of TTP patients, either on the basis of a constitutional deficiency in congenital cases or the presence of an autoantibody inhibitor in most sporadic adult-onset cases $^{7-10}$. These findings suggest that TTP may be triggered by accumulation of large, highly adhesive VWF multimers in the absence of physiologic processing by this VWF-cleaving protease. However, other studies have implicated platelet-aggregating proteins ${ }^{11,12}$ or endothelial injury ${ }^{13}$ as the underlying mechanism, and enhanced rather than decreased VWF proteolysis has been reported in some patients ${ }^{14}$. Although the VWF-cleaving protease has been partially purified $^{5,6}$, it seems to be present at relatively low levels in plasma and its identification at the sequence level has remained elusive.

\section{VWF-cleaving protease activity is a semidominant trait}

To further explore the molecular pathogenesis of TTP, we used a genetic approach to study the four pedigrees shown in Fig. 1. Activity of VWF-cleaving protease was measured in the plasma of the seven affected individuals, and was $2-7 \%$ of normal $(0.02-$ $0.07 \mathrm{U} \mathrm{ml}^{-1}$ ); none of the patients tested positive for inhibitors of the protease. Plasma levels of the protease in the parents of the affected individuals were $0.51-0.68 \mathrm{U} \mathrm{ml}^{-1}$, consistent with a heterozygous 
carrier state. Similarly, levels for at-risk siblings of the patients and parents fell into a bimodal distribution, with one peak consistent with carriers and the other indistinguishable from the normal distribution (Fig. 2). These results demonstrate that the protease activity assay used here reliably distinguishes between normal and carrier individuals in these families. This observation suggested that the plasma level of VWF-cleaving protease could be used as a phenotypic trait for linkage analysis to map the corresponding locus, providing considerably greater genetic power than would be available from analysis of the clinical phenotype alone.

\section{Gene for familial TTP maps to chromosome 9q34}

A genome-wide linkage scan was performed with 382 polymorphic microsatellite markers to analyse DNA from affected individuals and other informative family members (Fig. 1). Two-point linkage analysis with a recessive model gave a maximum lod (log likelihood ratio) score of 2.36 at $\theta=0.0$ for marker D9S164 on chromosome $9 \mathrm{q} 34$. A lod score of 5.63 at $\theta=0.0$ was obtained for the same marker with a codominant model. There was still substantial evidence of linkage to D9S164 when a codominant model with 95\% penetrance was used (lod score of 4.12 with $\theta=0.0$ at D9S164). Multipoint analysis for D9S164 and four flanking markers yielded a maximum lod score of 7.37 at marker D9S164. Genotypes for seven additional markers in this region ${ }^{15,16}$ allowed the gene to be placed in the $\sim 7$-centimorgan (cM) interval between markers D9S1863 and D9S1818 (Figs 1 and 3a). Analysis of new polymorphic markers (see Supplementary Information Table 3) designed using simple sequence-repeat data available from the Human Genome Project working draft (http://genome.ucsc.edu $)^{17}$ further narrowed the candidate interval to an $\sim 2.3$-megabase $(\mathrm{Mb})$ genomic segment between markers GL2-1 and D9S1818. In all but one case, carrier status as determined by haplotype analysis was consistent with the phenotypic designation according to plasma protease level. The exception, individual II2 in pedigree A, shares the affected haplotype of her brother (II4), but has a protease level of $0.8 \mathrm{U} \mathrm{ml}^{-1}$, which is borderline between the normal and carrier ranges.

\section{Gene for familial TTP encodes an ADAMTS metalloproteinase}

Analysis of the candidate interval using public genome databases (http://genome.ucsc.edu, http://www.ncbi.nlm.nih.gov) ${ }^{17}$ identified $\sim 20$ known or predicted genes (Fig. 3a). Initial attention focused on genes likely to encode a protease or protease cofactor. Ficolin 2 (FCN2) mapped to distal chromosome 9 but could not be identified in available BAC sequence from the candidate interval. However, in light of previous reports suggesting a proteaseassociated function for some ficolin family members ${ }^{18}$, and the possibility that FCN2 might lie in one of the three large genomic sequence gaps shown in Fig. 3a, the coding exons and intron-exon

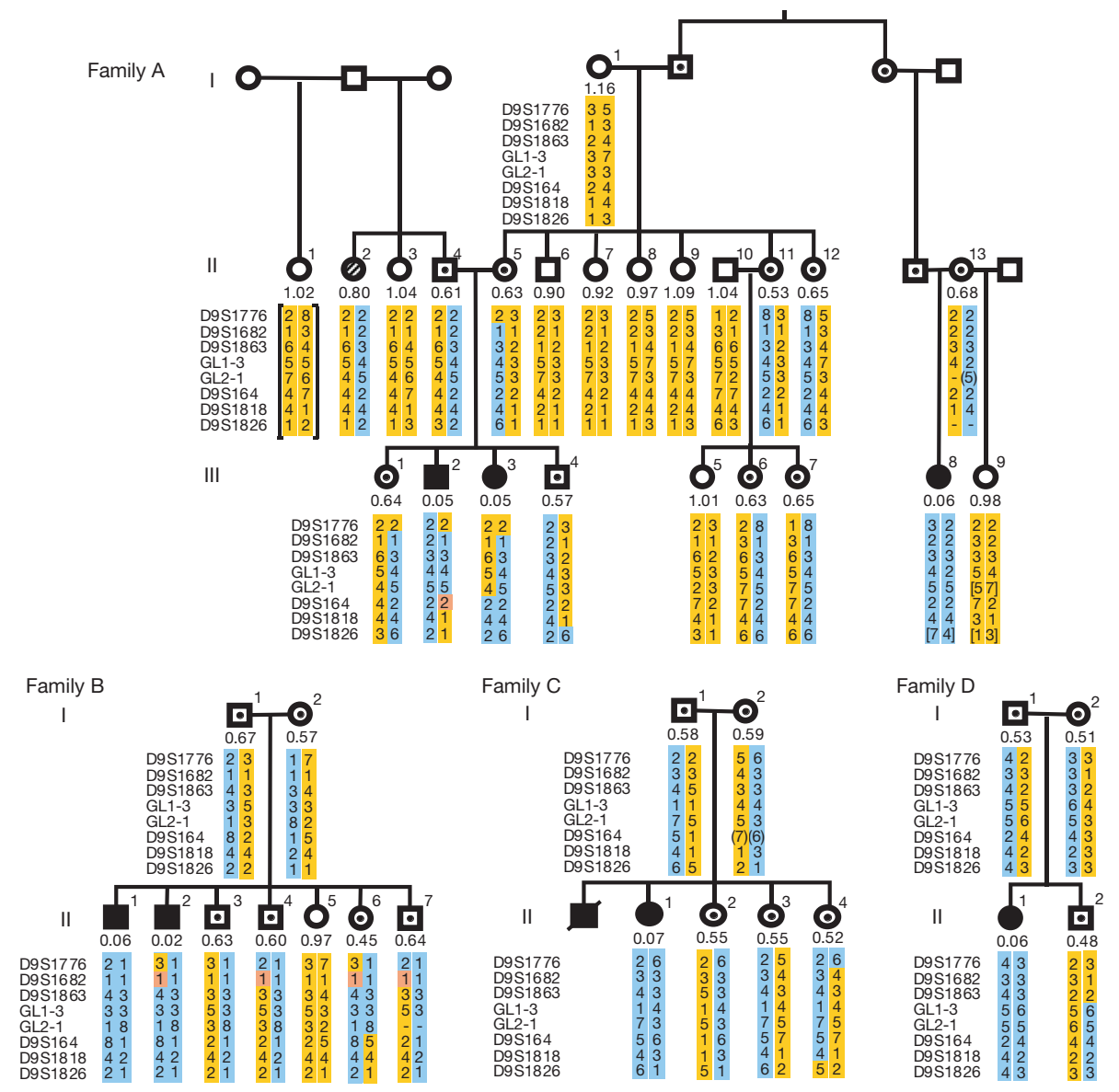

Figure 1 Pedigrees used for linkage analysis. VWF-cleaving protease levels (in units per millilitre) are shown beneath the symbol for each individual. Circles indicate females and squares indicate males. Affected individuals are indicated by solid symbols and carriers by symbols with dots. Individual All-2 was classified as phenotype unknown (hatched symbol) on the basis of an intermediate protease level (0.8). Seventeen markers (13 from the Genethon ${ }^{15}$ and Marshfield ${ }^{16}$ comprehensive genetic maps and four designed from sequence-repeat information available at the Human Genome Project working draft ${ }^{17}$ (see Supplementary Information Table 3)) were used for haplotype analysis. Only selected markers are shown. Chromosomes carrying affected alleles are blue and normal chromosomes are yellow. Areas where recombination cannot be definitively assigned are orange. Only recombination events between affected and unaffected chromosomes are shown. Inferred genotypes are indicated in parentheses, and genotypes of unknown phase in square brackets. Recombination events in individuals Alll-3 and Bll-6 place the responsible gene below marker GL2-1 and a recombination event in individual Alll-2 places the gene above marker D9S1818. 
boundaries of this gene were amplified by polymerase chain reaction (PCR) from patients' DNA and subjected to sequence analysis. No candidate mutations were identified. Two putative genes in the candidate interval, KIAA0605, an uncharacterized expressed sequence tag (EST) from a brain complementary DNA library ${ }^{19}$, and the predicted open reading frame C9ORF8, exhibited homology to the ADAMTS family of metalloproteinases, but seemed to lack the conserved protease catalytic domain. Partial DNA sequence analysis of exons and flanking intron sequences failed to identify any mutations in KIAA0605. However, the identification of several candidate missense mutations in the predicted exons of C9ORF8 led to further, more detailed analysis of this candidate gene. Through a combination of cDNA cloning, PCR with reverse transcription (RT-PCR), rapid amplification of cDNA ends (RACE) and genomic sequence analysis, the full-length ADAMTS13 cDNA sequence (GenBank accession number AF414401) and corresponding genomic structure were deduced (Fig. 3b) and found to encode a complete, potentially catalytically active ADAMTS protease.

Members of the ADAM (a disintegrin and metalloproteinase) family are membrane-anchored proteases with diverse functions. Known members include fertilins $\alpha$ and $\beta$, implicated in sperm-egg fusion, and the 'sheddases', such as TACE (TNF- $\alpha$ convertase), which mediate the shedding of cell-surface proteins ${ }^{20}$. Members of the ADAMTS family are distinguished from ADAMs by the presence of one or more thrombospondin-1-like (TSP1) domain(s) at the carboxy terminus, which are thought to mediate interactions with components of the extracellular matrix ${ }^{21-24}$. In addition, ADAMTS proteins lack the EGF repeat, transmembrane domain and cytoplasmic tail typically observed in ADAM metalloproteinases. Mutations in ADAMTS2 (procollagen N-proteinase), the only family
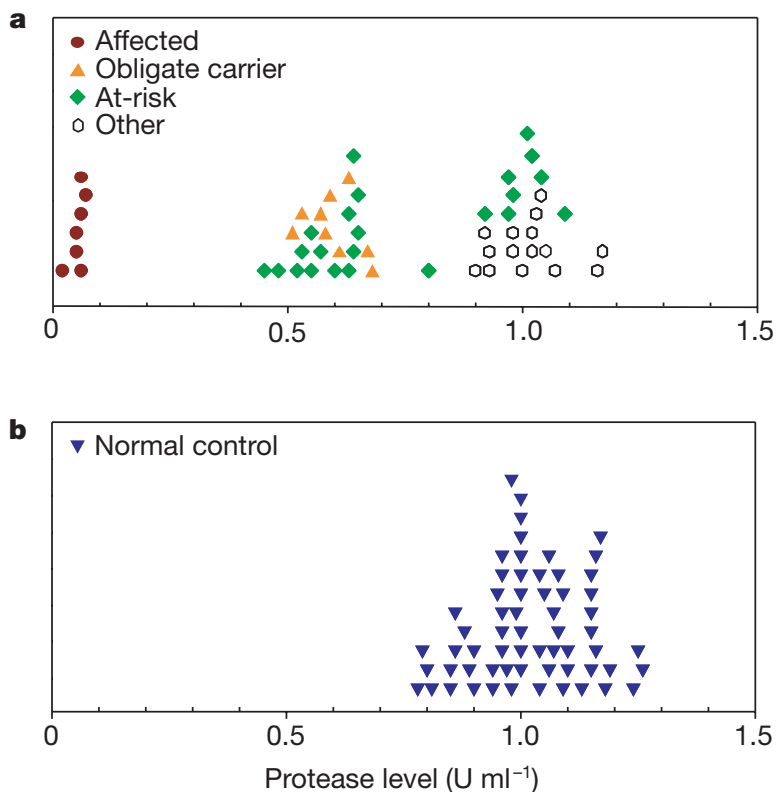

Figure 2 Plasma VWF-cleaving protease levels. a, Levels for all individuals shown in Fig. 1, as well as additional members of family A. b, Levels for 61 normal control individuals. Affected individuals are indicated by red circles, obligate carriers (parents of affected individuals) by orange triangles, other individuals at risk for inheriting an affected allele by green diamonds, and additional members of family A not at risk by open hexagons. Normal controls are shown as blue triangles. Levels for at-risk individuals (green diamonds in a) fall into a bimodal distribution, with one peak ranging from 0.45 to $0.68 \mathrm{U} \mathrm{ml}^{-1}$, consistent with carriers, and the other from 0.90 to $1.17 \mathrm{U} \mathrm{ml}^{-1}$,

indistinguishable from the normal distribution shown in $\mathbf{b}$. Not shown here, probands $E, F$ and $\mathrm{G}$ had VWF-cleaving protease activities of $0.05,0.04$ and $0.09 \mathrm{U} \mathrm{ml}^{-1}$, respectively, and no detectable inhibitor. The parents of proband $E$ had VWF-cleaving protease levels of 0.49 and $0.63 \mathrm{U} \mathrm{ml}^{-1}$ and the parents of proband $\mathrm{G}$ had levels of 0.53 and $0.68 \mathrm{U} \mathrm{ml}^{-1}$. member previously associated with a human genetic disease, result in the connective tissue disorder, Ehlers-Danlos syndrome type VII (ref. 25). Although ADAMTS1 mutations have not been identified in humans, genetically deficient mice exhibit growth retardation, adipose tissue abnormalities, and fibrotic changes throughout the genitourinary system, suggesting a critical importance for ADAMTS1 in organogenesis and tissue remodelling ${ }^{26}$. ADAMTS4 and ADAMTS5/11 (aggrecanases) cleave the core protein of the cartilage proteoglycan, aggrecan, and may be important in inflammatory joint disease ${ }^{27}$. Procollagen II has recently been identified as the substrate for ADAMTS3 (ref. 28). The function and protein substrates for the remaining ADAMTS family members are unknown.

The ADAMTS13 gene spans 29 exons encompassing $\sim 37 \mathrm{~kb}$ in the human genome and encodes a protein with 1,427 amino acids (Fig. 3b). Analysis of RT-PCR and cloned cDNA sequences provided evidence for alternative splicing of exon 17 (GenBank accession number AF414400), resulting in a frameshift that predicts a truncated 842-amino-acid form of the protein lacking the six C-terminal TSP1 repeats. Comparative analysis with draft mouse genomic sequences demonstrates a high degree of conservation throughout the coding exons and identifies an additional potential exon located between the current exons 22 and 23, which may indicate another splice isoform. These findings suggest the potential for differentially regulated alternative isoforms of ADAMTS13 with diverse biological functions in addition to the putative proteolytic processing of VWF. Alternative splicing has been observed for two other ADAMTS genes, ADAMTS2 (ref. 25) and ADAMTS9 (ref. 23), resulting in similar variation in the number of C-terminal TSP1 repeats.

The domain structure of ADAMTS13 is depicted at the bottom of Fig. 3b. A predicted signal peptide is followed by a short propeptide domain ending in a potential propeptide convertase cleavage site at amino acids 71-74 (RQRR), suggesting that proteolytic processing, either in the trans Golgi or at the cell surface, is required for activation. The protease domain that follows contains a perfect match for the HEXGHXXGXXHD (where $\mathrm{X}$ is any amino acid) consensus sequence of the extended catalytic site shared between snake venom metalloproteinases and ADAM family members ${ }^{20,21,29}$. The catalytic domain is followed by TSP1 and spacer domains characteristic of the ADAMTS family. An RGD sequence, present in only one other mature ADAMTS protein (ADAMTS2), is located immediately $\mathrm{C}$ terminal to the first TSP1 domain of ADAMTS13, suggesting a possible integrin interaction. The $\mathrm{C}$ terminus contains six additional TSP1 repeats, followed by a segment with homology to the CUB domain identified in a number of developmentally regulated proteins $s^{30}$. The previously reported inhibitor profile and metal cation dependence of the VWF-cleaving protease $e^{5,6,31}$ are consistent with its identity as an ADAMTS. The predicted, nonglycosylated relative molecular mass of ADAMTS13 is $154,000\left(M_{\mathrm{r}}\right.$ $154 \mathrm{~K})$, consistent with a previously estimated $M_{\mathrm{r}}$ of $200 \mathrm{~K}$ for partially purified VWF-cleaving protease ${ }^{5}$, although considerably smaller than the $300 \mathrm{~K} M_{\mathrm{r}}$ reported by others ${ }^{6}$.

Northern blot analysis detected an ADAMTS13 messenger RNA of $\sim 4.7-\mathrm{kb}$ specifically in the liver, with a truncated $(\sim 2.3 \mathrm{~kb})$ mRNA faintly visible in the placenta (Fig. 4a). These data suggest that plasma VWF-cleaving protease may be derived primarily from ADAMTS13 expression in the liver. The strong RT-PCR signal seen in the ovary, and variable expression in other tissues (Fig. 4b), may point to other potential functions for this protein. The absence of detectable transcripts in other highly vascular tissues such as the lung and heart may indicate that the vascular endothelium is not a primary site of ADAMTS13 expression.

\section{Mutations in ADAMTS13 cause familial TTP}

Analysis of DNA sequences identified mutations within the ADAMTS13 gene in all four of the pedigrees depicted in Fig. 1, as well as in three additional TTP patients not included in the original 
genome scan (families E-G, Table 1). The 12 mutations identified account for all but one of the 15 disease alleles expected in this set of patients (Table 1 and Fig. 5, and see Supplementary Information Figs 6 and 7). No recurrent mutation was observed, except in family A (Fig. 5). All three affected individuals in this family are homozygous for the same mutation carried on the same extended haplotype (data not shown), suggesting recent common ancestry. Although there is no known consanguinity, the parents of affected individuals AIII-2, AIII-3 and AIII- 8 are all from the same small village where the families have lived for several generations.

Two of the 12 identified mutations result in frameshifts (a 26-base pair (bp) deletion in exon 19 (Fig. 5) and a single $\mathrm{T}$ insertion in exon 27). A single splice mutation was identified in one family $(1584+5 \mathrm{G} \rightarrow \mathrm{A}$, family $\mathrm{G})$. This substitution markedly reduces or eliminates utilization of the normal intron 13 splice donor and activates a cryptic donor splice site at +70 , resulting in a 23 -codon insertion (see Supplementary Information Fig. 6). The remaining nine mutations all result in nonconservative amino-acid substitutions (Table 1) and all occur at positions that are perfectly conserved between the human and murine genes. H96D in the catalytic domain and $\mathrm{R} 398 \mathrm{H}$ in the first TSP1 domain both alter residues that are almost perfectly conserved among all known ADAMTS family members. All 12 of the ADAMTS13 gene mutations described above were excluded as common sequence polymorphisms by screening a large panel of unaffected chromosomes. Many single-nucleotide polymorphisms (SNPs) were also identified,

\begin{tabular}{|c|c|c|c|}
\hline Exon & Family & Nucleotide & Amino acid \\
\hline 3 & B & $286 C \rightarrow G$ & H96D \\
\hline 3 & $E$ & $304 \mathrm{C} \rightarrow \mathrm{T}$ & $\mathrm{R} 102 \mathrm{C}$ \\
\hline 6 & $E$ & $587 \mathrm{C} \rightarrow T$ & T196I \\
\hline 10 & $D$ & $1193 G \rightarrow A$ & $\mathrm{R} 398 \mathrm{H}$ \\
\hline 13 & C & $1582 A \rightarrow G$ & R528G \\
\hline 13 & G & $1584+5 G \rightarrow A$ & Splice \\
\hline 17 & $A$ & $2074 \mathrm{C} \rightarrow \mathrm{T}$ & R692C \\
\hline 19 & $\mathrm{~F}$ & $2376-2401 \Delta$ & Frameshift \\
\hline 22 & $B$ & $2851 \mathrm{~T} \rightarrow \mathrm{G}$ & C951G \\
\hline 24 & $\mathrm{D}$ & $3070 T \rightarrow G$ & C1024G \\
\hline 26 & $\mathrm{~F}$ & $3638 \mathrm{G} \rightarrow \mathrm{A}$ & C1213Y \\
\hline 27 & $\mathrm{C}$ & 3769-3770insT & Frameshift \\
\hline
\end{tabular}

Genomic DNA from patients was used to amplify exons and intron-exon boundaries of ADAMTS13. For mutations in families $\mathrm{A}-\mathrm{D}$, candidate mutations were confirmed in both parents. All mutations were confirmed as absent from $\sim 180$ control normal chromosomes by PCR, allele-specific oligonucleotide hybridization or restriction digest.

although only 7 of 25 result in amino-acid substitutions (see Supplementary Information Table 2).

The spectrum of ADAMTS13 mutations observed here is notable for the relative paucity of obvious null alleles. In addition, the single splice mutation and two frameshift mutations all occur in trans with a missense mutation on the other allele. These data suggest that complete deficiency of ADAMTS13 may be lethal, consistent with the low levels of residual VWF-cleaving protease activity observed in all ten deficient patients studied here $\left(0.02-0.09 \mathrm{U} \mathrm{ml}^{-1}\right)$.

a

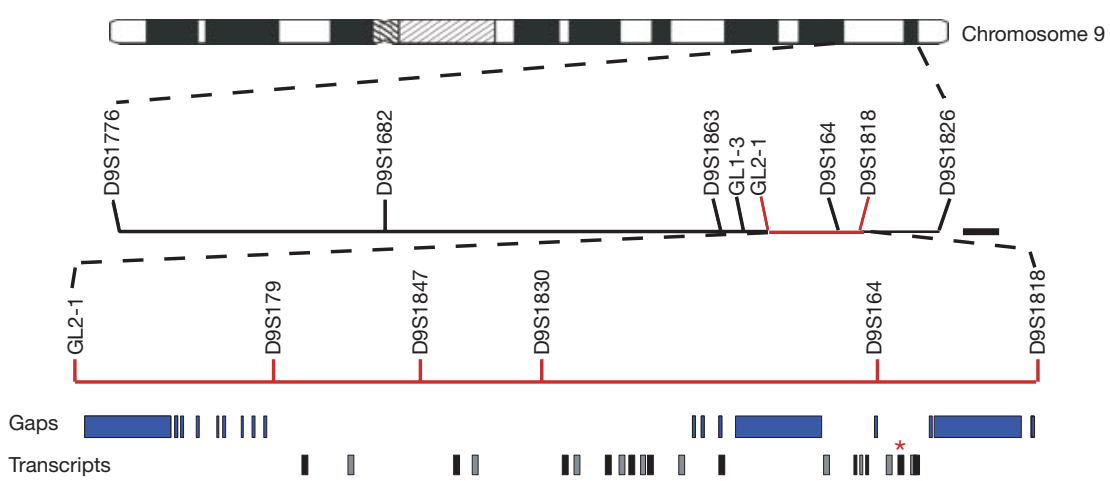

b

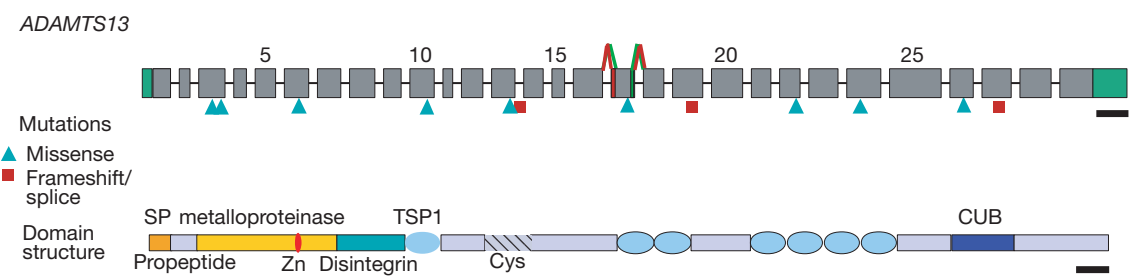

Figure 3 Identification of the ADAMTS13 gene. a, Physical map of chromosome 9 in the interval surrounding marker D9S164. The 2.3-Mb nonrecombinant interval identified in Fig. 1 is shown in red. Sequence gaps in the public genomic draft assembly are denoted by blue rectangles. Transcripts localized to this interval are depicted by alternating black and grey bars. The predicted gene C9ORF8 is indicated with a red asterisk and is located $\sim 32 \mathrm{~kb}$ telomeric to D9S164, on the basis of the current draft human genome sequence ${ }^{17}$. The reference bar represents $1 \mathrm{Mb}$. $\mathbf{b}$, Intron-exon and domain structures of ADAMTS13. The coding region is illustrated in grey and $5^{\prime}$ and $3^{\prime}$ untranslated regions in green. Intron sizes are not drawn to scale. Exon 1 of C90RF8 overlaps with a cluster of EST sequences (UniGene cluster Hs.149184), initially interpreted as predicting a large $5^{\prime}$ untranslated region. A segment of putative C9ORF8 coding sequence was used to identify two partial cDNA clones from human fetal liver, which were extended in both the $5^{\prime}$ and $3^{\prime}$ directions by RT-PCR and RACE. The assembled cDNA sequence corrected an error in the predicted boundaries of C9ORF8 exon 2, resulting in a continuous open reading frame including two exons upstream of the $5^{\prime}$ EST cluster, three new exons within the predicted intron 10 of C9ORF8 and six additional downstream exons encompassing a second hypothetical gene in this region, DKFZp434C2322 (Unigene cluster Hs.131433). Analysis of RT-PCR and cDNA sequences identified an alternatively spliced variant of exon 17 using both alternate donor and acceptor splice sites, as indicated. Mutations are depicted underneath the corresponding exons, with green triangles representing missense mutations and red squares representing frameshift and splice mutations. Scale bar, 200 nucleotides. The predicted domain structure of ADAMTS13 is shown at the bottom. The predicted signal peptide (predicted using SignalP version 1.1 (http://www.cbs.dtu.dk/services/SignalP)) is indicated in orange, the short propeptide in blue, the metalloproteinase domain in yellow, the disintegrin domain in green, and TSP1 domains as light blue ovals. The locations of the zinc-binding catalytic consensus within the metalloproteinase domain and the cysteine-rich region within the spacer domain are indicated. The CUB domain (dark blue) has not been identified in other ADAMTS family members. Scale bar, 50 amino acids. 


\section{Discussion}

The findings reported here identify genetic deficiency of ADAMTS13 as the underlying molecular mechanism responsible for most or all cases of familial TTP. This disorder seems to be identical to the congenital microangiopathic haemolytic anaemia also referred to as Schulman-Upshaw syndrome ${ }^{32}$ (OMIM number 276850). Although symptomatic anaemia and thrombocytopenia are usually present at birth, two of the patients studied here (proband G and BII-2) did not experience their first episodes of TTP until the ages of 4 and 8 years, respectively, despite clinical disease as neonates in siblings of both individuals. This highly variable age of onset, even within the same family, suggests a major contribution of other genetic and/or environmental modifying factors that remain to be identified. Previous studies have demonstrated antibody inhibitors directed against the VWF-cleaving protease in most adult TTP patients, suggesting an autoimmune aetiology ${ }^{7,10}$. However, the late onset of this disorder observed in two of the ten patients in our series raises the possibility that a subset of apparently sporadic adult-onset TTP may be due to unrecognized recessive inheritance of ADAMTS13 mutations.

The nearly perfect correlation of plasma VWF-cleaving protease activity with TTP carrier status that we observed was critical to the success of the genetic linkage analysis reported here. Although these data indicate that the plasma levels of this protease activity are very tightly regulated, to date no clinical phenotype has been detected in previously reported familial TTP carriers, or in any of the ADAMTS13-mutation heterozygotes identified here. However, the presence of a subtle phenotype in these individuals, such as increased susceptibility to thrombosis or to one or more of the 'acquired' forms of TTP, cannot be excluded.

Our data provide the first direct evidence of an aetiologic role for VWF-cleaving protease activity in the pathogenesis of TTP and identify the enzyme likely to be responsible for this activity: metalloproteinase ADAMTS13. Physiologic cleavage of VWF at the Tyr 842-Met 843 peptide bond produces the characteristic $176 \mathrm{~K}$ proteolytic fragment observed in normal plasma ${ }^{5,6,33}$ and increased susceptibility to this proteolytic cleavage seems to be

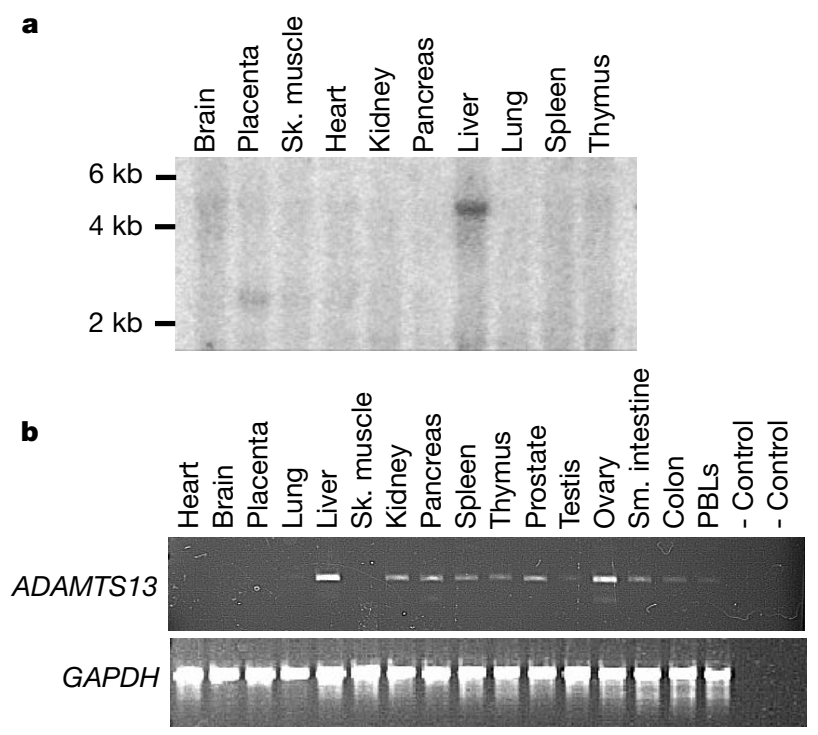

Figure 4 Northern and RT-PCR analysis of ADAMTS13. a, A northern blot of RNA from multiple human tissues was hybridized with a probe spanning exons 11-13 and part of exon 14. An $\sim 4$.7-kb message can be seen specifically in liver and a truncated $\sim 2.3-\mathrm{kb}$ message is faintly visible in placenta. $\mathbf{b}$, A panel of cDNAs derived from human tissues was screened by PCR for the presence of exons 10-14. Strong signals were seen in liver and ovary, with weak expression also evident in kidney, pancreas, spleen, thymus, prostate, testis, small (sm.) intestine and peripheral blood leukocytes (PBLs). No expression was detected in heart, brain, placenta, lung or skeletal (sk.) muscle. responsible for the loss of large VWF multimers central to the pathophysiology of type 2A von Willebrand disease (VWD) ${ }^{31,34}$.

Taken together with previous work from our group and others $^{4-7,10}$, our data strongly suggest that ADAMTS13 is the VWF-cleaving protease itself. However, we cannot exclude the possibility that ADAMTS13 acts on VWF indirectly, perhaps by activating another protease. In either case, our data are consistent with the hypothesis that accumulation of large, hyperactive VWF multimers in the absence of normal proteolytic processing triggers pathologic platelet aggregation and is the direct mechanism responsible for TTP. Alternatively, decreased VWF proteolysis could solely be a marker for the loss of ADAMTS13 activity. ADAMTS13 may also have important biological functions elsewhere in the

a T GGGT GT GG $\propto$ C C GC T GT GC OT GOGC
200

Control

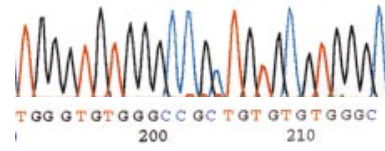

$2074 \mathrm{C} \rightarrow \mathrm{T}$

Family A

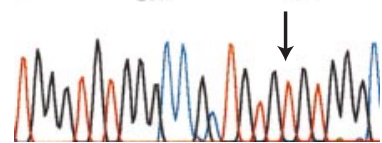

b

Control

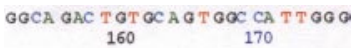

Control

$3638 \mathrm{G} \rightarrow \mathrm{A}$

Family $F$

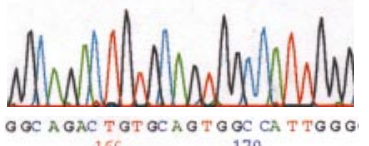

$16 \mathrm{C}$

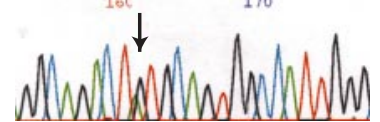

Control
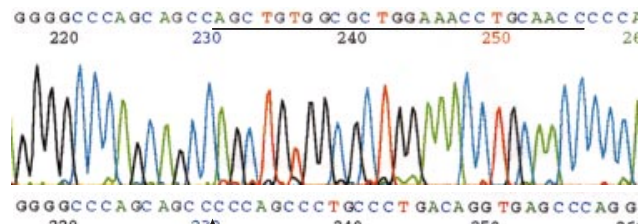

2376-2401ه

Family $\mathrm{F}$

220
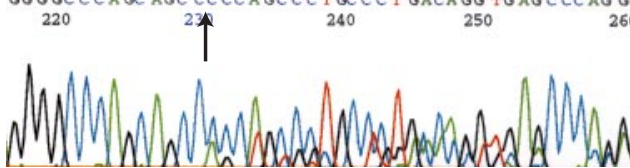

2376-2401s

Family $\mathrm{F}$

(cloned)

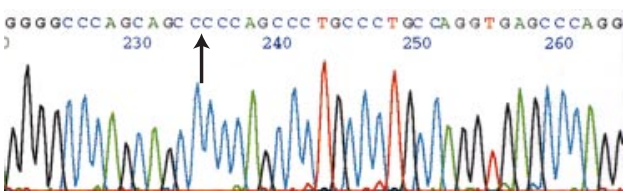

Figure 5 ADAMTS13 mutations in TTP patients. a, Sequence chromatograms of PCRamplified exon 17 from a control individual and an affected individual from family $A$. The arrow indicates the position of the $2074 \mathrm{C} \rightarrow \mathrm{T}$ (R692C) mutation. The single mutant $\mathrm{T}$ peak in the patient sequence chromatogram indicates homozygosity for this mutation. b. Sequence chromatograms of PCR-amplified exon 26 identify the heterozygous $3638 \mathrm{G} \rightarrow \mathrm{A}$ (C1213Y) mutation (arrowed), accounting for one of the mutant alleles in family F. c, Analysis of the second mutant allele in family F. Sequence chromatograms of PCR-amplified exon 19 from a normal control (top panel) and the cloned 2376-2401 $\Delta$ deletion allele (third panel) are shown. Sequence analysis of total PCR product from the patient (middle panel) demonstrates the superimposed normal and deletion allele sequences, indicating that the patient is heterozygous for this mutation. The underline in the control sequence indicates the 26-bp deletion with the arrows in the other traces indicating the start of the deletion. 
coagulation system or in the blood vessel wall, with loss of one or more of these activities providing the direct link to the pathogenesis of TTP. Future studies of ADAMTS13 function, both in vivo and in vitro, should answer these questions and provide insight into the complex biological processes regulating haemostasis and the maintenance of vascular integrity. Finally, the identification of ADAMTS13 deficiency as the cause of TTP also has major implications for the treatment of this important human disease, including the potential for production of recombinant ADAMTS13 as a safer and more effective replacement for current plasma-exchange therapy.

Note added in proof: Fujikawa et al. ${ }^{35}$ and Gerritsen et al. ${ }^{36}$ have reported purification and sequence analysis of the amino-terminal amino acids of human VWF-cleaving protease. These results confirm the identity of ADAMTS13 as the VWF-cleaving protease and its predicted processing by propeptide convertase cleavage.

\section{Methods}

\section{Subjects}

Patients included in this study were referred for evaluation of thrombocytopenia, haemolytic anaemia and schistocytes on blood smear. Probands for the four families (A-D) used in the linkage analysis all had a chronic relapsing course, responded to plasma infusion, and had the disorder as neonates or had a family member with such a disorder as a neonate. All three probands for kindreds $\mathrm{E}-\mathrm{G}$ also had microangiopathic haemolysis and/or thrombocytopenia noted soon after birth or in the early neonatal period (in the patient or an affected sibling), along with clinical response to plasma infusion.

Plasma samples were obtained from sodium citrate anticoagulated blood by centrifugation and saved at $-70^{\circ} \mathrm{C}$ as previously described ${ }^{7}$. Mononuclear cells were obtained from heparin anticoagulated blood by centrifugation on Ficoll-Hypaque, washed and transformed with Epstein-Barr virus. Informed consent was obtained from all individuals before sample collection (study protocol approved by the institutional review board).

\section{Multimer analysis and VWF-cleaving protease activity}

Analysis of VWF multimers was performed as previously described ${ }^{7}$. Ultra large VWF multimers were detected in the plasma of all TTP patients studied, with normal patterns observed in all other family members. For the measurement of VWF-cleaving protease activity, VWF treated with guanidine hydrochloride was used as the substrate. Protease activity was represented by the optical density of the dimer of the $176 \mathrm{~K}$ fragment generated from the VWF substrate ${ }^{7}$ and was expressed in units per millilitre, with the activity measured in pooled normal control plasma defined as $1 \mathrm{U} \mathrm{ml}^{-1}$. Protease activity is reported as the mean of two measurements for the parents of families E and G. All other samples were measured on at least three occasions, with the mean value reported. Assays for inhibitors of VWF-cleaving protease were performed as described ${ }^{37}$.

\section{Linkage analysis}

A genome-wide linkage screen was performed with 382 polymorphic microsatellite markers spaced an average of $10 \mathrm{cM}$ (panels 1-27 of the ABI Prism Linkage Mapping SetMD10 (Applied Biosystems)). We amplified 20 ng of genomic DNA with AmpliTaq Gold DNA polymerase (Applied Biosystems). PCR products were run on an ABI Prism 3700 DNA Analyzer and analysed with Genescan version 3.5NT and Genotyper version 3.6NT. Inspection of the pedigrees indicated an autosomal recessive mode of inheritance for TTP in this set of families. The frequency of the disease gene was assumed to be 1 per 10,000 chromosomes in the population. Population frequencies of the marker alleles were estimated from the genotyped individuals. Two-point lod scores were calculated with the program MLINK as implemented in the FASTLINK package version 3.0 (ref. 38), with an autosomal recessive model. A second series of analyses was performed with a codominant model to reflect the lowered enzyme levels of individuals who were assumed to be carriers of the disease gene. For the latter analysis, individuals were classified as affected (those with clinical diagnoses), carriers (those with protease levels in the range of $0.45-0.68 \mathrm{U} \mathrm{ml}^{-1}$ ) and unaffected (those with protease levels in the range of $0.9-1.17 \mathrm{U} \mathrm{ml}^{-1}$ ). One individual (AII-2), whose protease level was intermediate between the carrier and unaffected ranges, was classified as phenotype unknown. Penetrance was set at $100 \%$ for the recessive mode and codominant models. Consistency of the linkage evidence was evaluated by recomputing the lod score with $95 \%$ penetrance for the codominant model. Multipoint analyses were performed with the program VITESSE ${ }^{39}$, using the same two disease models and the five markers at or flanking the maximum two-point lod score (centromere-D9S1682-D9S290D9S164-D9S1826-D9S158-telomere). Order and distances between markers were determined with the ABI Prism Linkage Mapping Set-MD10 map information. To ensure the multipoint analyses were computationally feasible, the number of founders in pedigree A was reduced and less-informative individuals (II-1, $-2,-3$ and -13 , and III- 8 and -9 ) were pruned from the pedigree.

\section{Sequence analysis}

All exons and intron-exon boundaries of the predicted ADAMTS13 gene were amplified from patients' genomic DNA, with the exception of exon 7, which could not be amplified with multiple primer sets. Intron primers were selected with the Primer3 software packag (S. Rozen and H. J. Skaletsky, 1998; code available at http://www-genome.wi.mit.edu/ genome software/other/primer3.html) to allow for analysis of exon sequence as well as flanking donor and acceptor splice sites. (See Supplementary Information Table 4 for primer sequences.) We used $100 \mathrm{ng}$ of genomic DNA in a PCR reaction using either Platinum Taq DNA polymerase (Invitrogen), the Expand Long Template DNA polymerase mix (Roche) or the Advantage 2 DNA polymerase mix (Clontech). PCR products were either purified directly from the PCR reaction using the QIAquick PCR purification kit (Qiagen) or gel purified from low-melting agarose (Invitrogen) with the Wizard PCR Preps purification kit (Promega). Total cellular RNA from lymphoblast cell lines was prepared with Trizol (Invitrogen) and RT-PCR performed with the One-Step RT-PCR kit (Invitrogen), according to the manufacturer's instructions. Sequencing reactions were performed by the University of Michigan DNA Sequencing Core. Selected PCR products were subcloned into a pCR-TOPO plasmid (Invitrogen) for further sequence analysis.

\section{Allele-specific oligonucleotide hybridization}

Individual exons were amplified from 92 unrelated control individuals. For allele-specific oligonucleotide hybridization, PCR products were spotted onto nitrocellulose membranes by a dot-blot apparatus (Invitrogen). Fifteen-base oligonucleotides corresponding to wild-type or mutant alleles were end-labelled with $\gamma^{3}{ }^{32} \mathrm{P}$-ATP by T4 polynucleotide kinase (New England Biolabs). Hybridization was performed in ExpressHyb solution (Clontech) at $37^{\circ} \mathrm{C}$. Blots were washed in $5 \times$ SSPE, $0.1 \%$ SDS at a temperature determined empirically for each oligonucleotide. Restriction-digested PCR products were analysed on a 3\% NuSieve GTG agarose (BioWhittaker), 1\% agarose (Invitrogen) gel.

\section{RT-PCR and northern blot analysis}

RT-PCR on patient RNA and PCR on cDNA obtained from a Multiple Tissue cDNA panel (Clontech) were performed using primers $5^{\prime}$-CAGTGCAACAACCCCAGAC- $3^{\prime}$ and 5'-GGCACCTGTCCCATACCTG-3', which amplify cDNA nucleotides 1,228-1,636 (numbered from the initiator ATG as +1 ). A FirstChoice Human Northern Blot (Ambion) was screened with a probe generated by random priming using the Rediprime II kit (Amersham) of a PCR product amplified from a human MOLT4 T-cell cDNA library (generated as previously described ${ }^{40}$ ) with the above primers. Hybridization was performed in ExpressHyb solution (Clontech) according to the manufacturer's specifications The final wash step was performed in $0.1 \times \mathrm{SSC}, 0.1 \% \mathrm{SDS}$ at $50^{\circ} \mathrm{C}$.

\section{Isolation of cDNA}

A human fetal liver cDNA library in $\lambda$ gt10 (Clontech) was screened with the northern probe described above. Two overlapping cDNA clones were obtained, spanning exons 5-14 and 8-20 of the predicted ADAMTS13 cDNA sequence. Phage DNA was purified with a Nucleobond lambda midi kit (Clontech), digested with EcoRI (New England Biolabs) and subcloned into pBSII-SK+ (Stratagene). We performed 5' RACE on FirstChoice RACE-Ready human liver cDNA (Ambion). Additional 5' RACE was performed with the 5'/3' RACE kit (Roche) on human liver polyA+ RNA (Ambion). Marathon-Ready human liver cDNA (Clontech) was used for 3' RACE and for an internal RT-PCR, the latter to amplify a product spanning cDNA nucleotides 1,552-2,627. RACE and RT-PCR products were cloned into a pCR-TOPO vector (Invitrogen) and individual clones were subjected to sequence analysis. Nucleotides bridging $5^{\prime}$ RACE and cDNA clones (cDNA nucleotides 456-534) were obtained from the C9ORF8 EST cluster (UniGene cluster Hs.149184). Exon-intron boundaries and sequence accuracy were verified against available draft human sequence at http://www.ncbi.nlm.nih.gov ${ }^{17}$.

Received 30 July; accepted 6 September 2001.

1. Cines, D. B., Konkle, B. A. \& Furlan, M. Thrombotic thrombocytopenic purpura: a paradigm shift? Thromb. Haemost. 84, 528-535 (2000).

2. George, J. N. How I treat patients with thrombotic thrombocytopenic purpura-hemolytic uremic syndrome. Blood 96, 1223-1229 (2000).

3. Rock, G. A. et al. Comparison of plasma exchange with plasma infusion in the treatment of thrombotic thrombocytopenic purpura. N. Engl. J. Med. 325, 393-397 (1991).

4. Moake, J. L. et al. Unusually large plasma factor VIII: von Willebrand factor multimers in chronic relapsing thrombotic thrombocytopenic purpura. N. Engl. J. Med. 307, 1432-1435 (1982).

5. Tsai, H. M. Physiologic cleavage of von Willebrand factor by a plasma protease is dependent on its conformation and requires calcium ion. Blood 87, 4235-4244 (1996).

6. Furlan, M., Robles, R. \& Lämmle, B. Partial purification and characterization of a protease from human plasma cleaving von Willebrand factor to fragments produced by in vivo proteolysis. Blood $\mathbf{8 7}$, 4223-4234 (1996).

7. Tsai, H.-M. \& Lian, E. C. Y. Antibodies to von Willebrand factor-cleaving protease in acute thrombotic thrombocytopenic purpura. N. Engl. J. Med. 339, 1585-1594 (1998).

8. Tsai, H.-M., Rice, L., Sarode, R., Chow, T. W. \& Moake, J. L. Antibody inhibitors to von Willebrand factor metalloproteinase and increased binding of von Willebrand factor to platelets in ticlopidineassociated thrombotic thrombocytopenic purpura. Ann. Intern. Med. 132, 794-799 (2000).

9. Bennett, C. L. et al. Thrombotic thrombocytopenic purpura associated with clopidogrel. N. Engl. J. Med. 342, 1773-1777 (2000)

10. Furlan, M. et al. von Willebrand factor-cleaving protease in thrombotic thrombocytopenic purpura and the hemolytic-uremic syndrome. N. Engl. J. Med. 339, 1578-1584 (1998).

11. Burns, E. R. \& Zucker-Franklin, D. Pathologic effects of plasma from patients with thrombotic thrombocytopenic purpura on platelets and cultured vascular endothelial cells. Blood 60, 1030-1037 (1982).

12. Siddiqui, F. A. \& Lian, E. C. Novel platelet-agglutinating protein from a thrombotic thrombocytopenic purpura plasma. J. Clin. Invest 76, 1330-1337 (1985). 
13. Mitra, D. et al. Thrombotic thrombocytopenic purpura and sporadic hemolytic-uremic syndrome plasmas induce apoptosis in restricted lineages of human microvascular endothelial cells. Blood 89 1224-1234 (1997)

14. Mannucci, P. M. et al. Enhanced proteolysis of plasma von Willebrand factor in thrombotic thrombocytopenic purpura and the hemolytic uremic syndrome. Blood 74, 978-983 (1989).

15. Dib, C. et al. A comprehensive genetic map of the human genome based on 5,264 microsatellites. Nature 380, 152-154 (1996).

16. Broman, K. W., Murray, J. C., Sheffield, V. C., White, R. L. \& Weber, J. L. Comprehensive human genetic maps: individual and sex-specific variation in recombination. Am. J. Hum. Genet. 63, 861-869 (1998).

17. Lander, E. S. et al. Initial sequencing and analysis of the human genome. Nature 409, 860-921 (2001).

18. Matsushita, M. \& Fujita, T. Ficolins and the lectin complement pathway. Immunol. Rev. 180, 78-85 (2001).

19. Nagase, T. et al. Prediction of the coding sequences of unidentified human genes. IX. The complete sequences of 100 new cDNA clones from brain which can code for large proteins in vitro. DNA Res. 5, 31-39 (1998).

20. Blobel, C. P. Metalloprotease-disintegrins: links to cell adhesion and cleavage of TNF $\alpha$ and Notch. Cell 90, 589-592 (1997)

21. Kaushal, G. P. \& Shah, S. V. The new kids on the block: ADAMTSs, potentially multifunctional metalloproteinases of the ADAM family. J. Clin. Invest. 105, 1335-1337 (2000).

22. Hurskainen, T. L., Hirohata, S., Seldin, M. F. \& Apte, S. S. ADAM-TS5, ADAM-TS6, and ADAM-TS7, novel members of a new family of zinc metalloproteases. General features and genomic distribution of the ADAM-TS family. J. Biol. Chem. 274, 25555-25563 (1999).

23. Tang, B. L. ADAMTS: a novel family of extracellular matrix proteases. Int. J. Biochem. Cell Biol. 33, 33 44 (2001).

24. Tang, B. L. \& Hong, W. ADAMTS: a novel family of proteases with an ADAM protease domain and thrombospondin 1 repeats. FEBS Lett. 445, 223-225 (1999).

25. Colige, A. et al. Human Ehlers-Danlos syndrome type VII C and bovine dermatosparaxis are caused by mutations in the procollagen I N-proteinase gene. Am. J. Hum. Genet. 65, 308-317 (1999).

26. Shindo, T. et al. ADAMTS-1: a metalloproteinase-disintegrin essential for normal growth, fertility, and organ morphology and function. J. Clin. Invest. 105, 1345-1352 (2000).

27. Tortorella, M. D. et al. Purification and cloning of aggrecanase-1: a member of the ADAMTS family of proteins. Science 284, 1664-1666 (1999)

28. Fernandes, R. J. et al. Procollagen II amino propeptide processing by ADAMTS-3. Insights on dermatosparaxis. J. Biol. Chem. 276, 31502-31509 (2001).

29. Kuno, K. et al. Molecular cloning of a gene encoding a new type of metalloproteinase-disintegrin family protein with thrombospondin motifs as an inflammation associated gene. J. Biol. Chem. 272, 556-562 (1997).
30. Bork, P. \& Beckmann, G. The CUB domain. A widespread module in developmentally regulated proteins. J. Mol. Biol. 231, 539-545 (1993).

31. Tsai, H.-M. et al. Proteolytic cleavage of recombinant type $2 \mathrm{~A}$ von Willebrand factor mutants R834W and R834Q: Inhibition by doxycycline and by monoclonal antibody VP-1. Blood 89, 1954-1962 (1997).

32. Upshaw, J. D. Jr Congenital deficiency of a factor in normal plasma that reverses microangiopathic hemolysis and thrombocytopenia. N. Engl. J. Med. 298, 1350-1352 (1978).

33. Zimmerman, T. S., Dent, J. A., Ruggeri, Z. M. \& Hannini, L. H. Subunit composition of plasma von Willebrand factor. J. Clin. Invest. 77, 947-951 (1986).

34. Lyons, S. E., Bruck, M. E., Bowie, E. J. W. \& Ginsburg, D. Impaired intracellular transport produced by a subset of type IIA von Willebrand disease mutations. J. Biol. Chem. 267, 4424-4430 (1992).

35. Fujikawa, K., Suzuki, H., McMullen, B. \& Chung, D. Purification of human von Willebrand factorcleaving protease and its identification as a new member of the metalloproteinase family. Blood $\mathbf{9 8}$, 1662-1666 (2001)

36. Gerritsen, H. E., Robles, R., Lämmle, B. \& Furlan, M. Partial amino acid sequence of purified von Willebrand factor-cleaving protease. Blood 98, 1654-1661 (2001).

37. Tsai, H. M., Li, A. \& Rock, G. Inhibitors of von Willebrand factor-cleaving protease in thrombotic thrombocytopenic purpura. Clin. Lab. 47, 387-392 (2001).

38. Schaffer, A. A., Gupta, S. K., Shriram, K. \& Cottingham, R. W. Jr Avoiding recomputation in linkage analysis. Hum. Hered. 44, 225-237 (1994).

39. O'Connell, J. R. \& Weeks, D. E. The VITESSE algorithm for rapid exact multilocus linkage analysis via genotype set-recoding and fuzzy inheritance. Nature Genet. 11, 402-408 (1995).

40. Ginsburg, D. et al. Human von Willebrand factor (vWF): isolation of complementary DNA (cDNA) clones and chromosomal localization. Science 228, 1401-1406 (1985).

Supplementary information is available on Nature's World-Wide Web site (http://www.nature.com) or as paper copy from the London editorial office of Nature.

\section{Acknowledgements}

We thank S. J. Weiss for comments on the manuscript; R. L. Nagel, I. I. Sussman, T.-P. Lee, J. Ott and J. E. Sadler for advice and encouragement; and A. Li and S. K. Uniacke for technical assistance. This work was supported in part by grants from the National Institutes of Health to H.-M.T., D.G., T.F. and W.C.N.; D.G. is an investigator of the Howard Hughes Medical Institute.

Correspondence and requests for materials should be addressed to D.G. (e-mail: ginsburg@umich.edu). Original cDNA and sequence-tagged site sequences reported here are available from GenBank under accession numbers AF414400, AF414401, G73162, G73163, G73164 and G73165. 